\title{
Vaginal delivery in SARS-CoV-2-infected pregnant women in Israel: a multicenter prospective analysis
}

\author{
Amihai Rottenstreich ${ }^{1}$ (1) - Abraham Tsur ${ }^{2} \cdot$ Nava Braverman $^{1}$. Doron Kabiri ${ }^{1}$. Shay Porat ${ }^{1} \cdot$ Shmuel Benenson $^{3}$. \\ Yonatan Oster ${ }^{3}$. Hadas Allouche Kam ${ }^{1}$. Asnat Walfisch ${ }^{1}$. Yossi Bart ${ }^{2} \cdot$ Raanan Meyer $^{2}$ - Shirlee Jaffe Lifshitz ${ }^{4}$. \\ Uri Amikam $^{5}$. Tal Biron-Shental ${ }^{6}$. Gal Cohen ${ }^{6}$. Yael Sciaky-Tamir ${ }^{7} \cdot$ Inbar Ben Shachar ${ }^{7} \cdot$ Yoav Yinon $^{2}$. \\ Benjamin Reubinoff'
}

Received: 27 August 2020 / Accepted: 17 October 2020 / Published online: 29 October 2020

(c) Springer-Verlag GmbH Germany, part of Springer Nature 2020

\begin{abstract}
Key Message Among SARS-CoV-2-infected mothers, vaginal delivery rates were high and associated with favorable outcomes with no cases of neonatal COVID-19.

Purpose To investigate the mode of delivery and its impact on immediate neonatal outcome in SARS-CoV-2-infected women. Methods A prospective study following pregnant women diagnosed with COVID-19 who delivered between March 15th and July 4th in seven university affiliated hospitals in Israel.

Results A total of 52 women with a confirmed diagnosis of COVID-19 delivered in the participating centers during the study period. The median gestational age at the time of delivery was 38 weeks, with $16(30.8 \%)$ cases complicated by spontaneous preterm birth. Forty-three women (82.7\%) underwent a trial of labor. The remaining 9 women underwent pre-labor cesarean delivery mostly due to obstetric indications, whereas one woman with a critical COVID-19 course underwent urgent cesarean delivery due to maternal deterioration. Among those who underwent a trial of labor $(n=43), 39(90.7 \%)$ delivered vaginally, whereas 4 (9.3\%) cases resulted in cesarean delivery. Neonatal RT-PCR nasopharyngeal swabs tested negative in all cases, and none of the infants developed pneumonia. No maternal and neonatal deaths were encountered.

Conclusions In this prospective study among SARS-CoV-2-infected mothers, vaginal delivery rates were high and associated with favorable outcomes with no cases of neonatal COVID-19. Our findings underscore that delivery management among SARS-CoV-2-infected mothers should be based on obstetric indications and may potentially reduce the high rates of cesarean delivery previously reported in this setting.
\end{abstract}

Keywords Pregnancy $\cdot$ Delivery $\cdot$ COVID-19 $\cdot$ Vaginal $\cdot$ Outcomes

Amihai Rottenstreich and Abraham Tsur equal contribution.

Yoav Yinon

yoav.yinon27@gmail.com

Benjamin Reubinoff

benr@hadassah.org.il

1 Department of Obstetrics and Gynecology, HadassahHebrew University Medical Center, POB 12000,

91120 Jerusalem, Israel

2 Department of Obstetrics and Gynecology, Sheba Medical Center, Sackler Faculty of Medicine, Tel-Aviv University, Tel-Hashomer, 52621 Ramat Gan, Israel

3 Department of Clinical Microbiology and Infectious Diseases, Hadassah-Hebrew University Medical Center, Jerusalem, Israel
4 Department of Obstetrics and Gynecology, Mayenei Hayeshua Medical Center, Sackler Faculty of Medicine, Tel-Aviv University, Ramat Gan, Israel

5 Department of Obstetrics and Gynecology, Lis Maternity Hospital, Sourasky Medical Center, Tel-Aviv, Sackler Faculty of Medicine, Tel-Aviv University, Tel Aviv, Israel

6 Department of Obstetrics and Gynecology, Meir Medical Center, Sackler Faculty of Medicine, Tel-Aviv University, Kfar Saba, Israel

7 Department of Obstetrics and Gynecology, Ziv Medical Center, Safed Azrieli Faculty of Medicine, Bar-Ilan University, Safed, Israel 


\section{Introduction}

The World Health Organization (WHO) named the new coronavirus (SARS-CoV-2) disease Coronavirus Disease 19 (COVID-19) and has declared that COVID-19 is a pandemic [1]. The perinatal outcomes of pregnant women infected with SARS-CoV-2 are currently not well established. While some reports provided reassuring data $[2,3]$, others have shown various adverse neonatal outcomes including preterm delivery, respiratory morbidity, thrombocytopenia, liver injury and even death, raising a concern in this regard [4]. Moreover, the possibility of vertical transmission is still under a major debate [4-8]. Vertical transmission could potentially occur during a trial of vaginal delivery, through neonatal contact with vaginal and rectal fluids or blood, as is already well known for other pathogens (e.g., group B streptococcus, human papilloma virus, human immunodeficiency virus) $[9,10]$. Current professional society guidelines recommend that the mode of delivery among women-tested positive for COVID-19 should be based upon obstetric indications [11, 12]. However, the rate of cesarean delivery in reported cases was relatively high $[13,14]$, reflecting the uncertainty in this regard.

Given the inconclusive evidence in current literature and the high clinical relevance, we aimed to investigate the mode of delivery and its impact on immediate neonatal outcome in SARS-CoV-2-infected women in Israel.

\section{Materials and methods}

\section{Patients}

A prospective study following pregnant women diagnosed with COVID-19 who delivered between March 15th and July 4th in seven university affiliated hospitals in Israel, located throughout the country, and serving heterogeneous, multicultural, and multinational population from both heavily populated urban and rural areas. Inclusion criteria included pregnant women with a confirmed diagnosis of COVID-19 prior to or within $48 \mathrm{~h}$ after delivery. Those diagnosed at the puerperium beyond this time period or those with a previous confirmed COVID-19 diagnosis that has resolved (as confirmed by RT-PCR nasopharyngeal swabs) prior to delivery were excluded. All women were treated according to the national guidelines for COVID19 in pregnancy, and treatment was individualized based upon signs, symptoms, and laboratory and imaging findings. Timing and mode of delivery in all participating hospitals were determined primarily on the basis of obstetric indications. Women with a confirmed diagnosis of COVID-19 prior to delivery, and those with suggestive symptoms of the disease or recent close contact with individuals with a confirmed diagnosis, were delivered in a negative-pressure isolation room, with strict infection protection measures for all attending staff throughout the course of delivery. Neonates of mothers with known or suspected COVID-19 were isolated until maternal COVID-19 diagnosis was ruled out, or if maternal infection was confirmed until neonatal RT-PCR nasopharyngeal swabs returned negative (twice, at 24 and $48 \mathrm{~h}$ after delivery). Direct breastfeeding in those with known or suspected COVID-19 was discouraged, while expressing milk under sterile conditions was allowed.

\section{Data collection}

For the purpose of this study, we abstracted maternal hospital admission records, delivery charts, surgical reports, and discharge letters from the electronic medical record databases of all participating centers. Data regarding maternal and pregnancy characteristics, as well as delivery and neonatal outcomes, were extracted. Data were captured using REDCap database. Symptoms were assessed according to patients' self-reported questionnaire. Disease severity was classified as mild, severe, or critical according to $\mathrm{Wu}$ and McGoogan's Classification [15].

\section{Statistical analysis}

Data are described as proportions for categorical variables and as medians and interquartile ranges for continuous variables without a normal distribution. The data were analyzed using Software Package for Statistics and Simulation (IBM SPSS version 24, IBM Corp, Armonk, NY).

\section{Results}

A total of 52 women with a confirmed diagnosis of COVID19 delivered in the participating centers during the study period. The median maternal age was 28 [IQR 24-35] years; $9(17.3 \%)$ were nulliparous. All women had a singleton gestation. Diagnosis of COVID-19 was made prior to hospital admission in $21(40.4 \%)$ cases, in the delivery ward in 25 $(48.1 \%)$ cases, and in $6(11.5 \%)$ cases the diagnosis was made within $48 \mathrm{~h}$ following delivery during the stay at the maternity ward. COVID-19 testing was performed in 21 (40.4\%) cases due to the presence of symptoms, while in the remaining parturients, it was made either as part of routine testing of all parturients $(n=18)$ or due to presumed exposure to known confirmed cases of COVID-19 $(n=13)$ (Table 1). Most cases were classified as mild $(n=26,50.0 \%)$, 
Table 1 Baseline and laboratory characteristics of parturients diagnosed with COVID-19

\begin{tabular}{ll}
\hline Characteristics & $n=52$ \\
\hline Age (years) & $28[24-35](30)$ \\
$>35$ years & $16(30.8 \%)$ \\
Parity & $2[1-6](3)$ \\
Nulliparous & $9(17.3 \%)$ \\
Reasons for SARS-CoV-2 testing & \\
Reported symptoms & $21(40.4 \%)$ \\
Routine screening & $18(34.7 \%)$ \\
Presumed exposure to affected individuals & $13(25.0 \%)$ \\
Symptomatic & $27(51.9 \%)$ \\
Fever & $11(21.2 \%)$ \\
Cough & $14(26.9 \%)$ \\
Dyspnea & $6(11.5 \%)$ \\
Sore throat & $4(7.7 \%)$ \\
Gastrointestinal symptoms & $4(7.7 \%)$ \\
Loss of taste/smell & $5(9.6 \%)$ \\
Platelet count (X 10 9 /L) & $191[156-240](197)$ \\
White blood cell count (X 10 9 /L) & $9.8[8.0-11.8](10.4)$ \\
Hemoglobin (g/dL) & $12.5[11.9-13.3](12.4)$ \\
Abnormal liver enzymes* & $6(11.5 \%)$ \\
C-reactive protein (mg/L) & $17.1[3.0-29.5](31.3)$ \\
\hline
\end{tabular}

All continuous variables are expressed as medians [interquartile range] (means)

*Aspartate aminotransferase or alanine aminotransferase higher than two-fold the upper limit of normal range ( $>35 \mathrm{IU} / \mathrm{L})$

${ }^{\dagger}$ Normal range for C-reactive protein $<5 \mathrm{mg} / \mathrm{L}$

none as severe, and one woman had a critical respiratory illness. The remaining 25 (48.1\%) women remained asymptomatic throughout their disease course,

The median gestational age at the time of delivery was 38 weeks, with 17 (32.7\%) cases complicated by preterm birth, mostly (16/17) following spontaneous onset of labor; of them, 6 occurred at less than 34 weeks of gestation. The rate of gestational hypertensive disorders was $13.4 \%$ (7/52). The rate of comorbidities was $9.6 \%$ (5/52) including hypothyroidism $(n=2)$, and rheumatic arthritis, rheumatic heart disease, and protein $\mathrm{S}$ deficiency in one case each.

Forty-three women $(82.7 \%)$ underwent a trial of labor. The remaining 9 women underwent pre-labor cesarean delivery mostly due to obstetric indications (previous cesarean deliveries, $n=5$; malpresentation, $n=3$ ), whereas one woman with a critical COVID-19 course underwent urgent cesarean delivery due to maternal deterioration. Among those who underwent a trial of labor $(n=43), 39(90.7 \%)$ delivered vaginally, whereas $4(9.3 \%)$ cases resulted in cesarean delivery (fetal distress, $n=3$; arrest of the second stage of labor, $n=1$ ) (Table 2).

Median neonatal birthweight was 3280 [IQR 2927-3606] grams. One very-preterm newborn had a 5-min Apgar score
Table 2 Pregnancy and delivery characteristics and outcomes among parturients diagnosed with COVID-19

\begin{tabular}{ll}
\hline Characteristics & $n=52$ \\
\hline Gestational age at delivery & $38[36-40](38)$ \\
$<37$ weeks & $17(32.7 \%)$ \\
<34 weeks & $9(17.3 \%)$ \\
Gestation diabetes mellitus & $6(11.5 \%)$ \\
Gestational hypertensive disorders & $7(13.4 \%)$ \\
Maternal complications & \\
Need for mechanical intubation & $1(1.9 \%)$ \\
Intensive-care unit admission & $1(1.9 \%)$ \\
Venous thromboembolism & $1(1.9 \%)$ \\
HELLP syndrome & $1(1.9 \%)$ \\
Spontaneous onset of labor & $33(63.5 \%)$ \\
Mode of anesthesia & \\
None & $15(28.8 \%)$ \\
General & $2(3.8 \%)$ \\
Regional & $35(67.3 \%)$ \\
Mode of delivery & \\
Vaginal & $39(75.0 \%)$ \\
Pre-labor cesarean & $9(17.3 \%)$ \\
In-labor cesarean & $4(7.7 \%)$ \\
Oxytocin use* & $18(41.9 \%)$ \\
Intrapartum fever* & $3(7.0 \%)$ \\
First-stage duration of labor (minutes) & $112[51-221](163)$ \\
Second-stage duration of labor (minutes) & $12[7-40](32)$ \\
Postpartum hemorrhage & $6(11.5 \%)$ \\
Length of hospitalization (days) & $4[3-7](6)$ \\
Neonatal outcomes & \\
Birthweight (grams) & $3280[2927-3606](3140)$ \\
5-min Apgar score <8 & $1(1.9 \%)$ \\
NICU admission & $6(11.5 \%)$ \\
RDS & $6(11.5 \%)$ \\
TTN & $1(1.9 \%)$ \\
Hypoglycemia & $4(9.3 \%)$ \\
Hyperbilirubinemia & $5.6 \%)$ \\
\hline
\end{tabular}

All continuous variables are expressed as medians [interquartile range] (means)

HELLP hemolysis elevated liver enzymes, and low platelets, $R D S$ respiratory distress syndrome, TTN transient tachypnea of the newborn

*The denominator is the total number of women undergoing a trial of vaginal delivery $(n=43)$

of 7; all other neonates had 5-min Apgar scores higher than 7. No congenital abnormalities were encountered, except for one neonate with single umbilical artery.

Neonatal RT-PCR nasopharyngeal swabs tested negative in all cases, and none of the infants developed pneumonia. No maternal and neonatal deaths were encountered. Six (11.5\%) preterm neonates were complicated by respiratory distress syndrome and were hospitalized in neonatal intensive-care units. Neonatal complications including sepsis, 
intraventricular hemorrhage, asphyxia, and necrotizing enterocolitis were not observed (Table 2).

\section{Discussion}

In this study, we examined the obstetric outcomes of 52 parturients affected by COVID-19. Vaginal delivery rate was high, and all neonates were tested negative for SARS-CoV-2.

The current guidelines of various professional Obstetrics and Gynecology Societies recommend that the mode of delivery among SARS-CoV-2-infected parturients should be determined on the basis of obstetric indications $[11,12]$. However, previous studies on COVID-19 in pregnancy reported remarkably high rates of cesarean delivery [13, $14,16]$. This may be related, at least in part, to concerns regarding the possibility of vertical transmission. Emerging evidence, while limited, suggests that vertical transmission is possible [5-8]. In the current study, we report a relatively high rate of vaginal delivery with no cases of neonatal COVID-19. Therefore, our findings support the aforementioned recommendations and provide further evidence regarding the safety of vaginal delivery in this setting.

The rate of preterm birth in the current cohort was substantial, with one third of women delivering prematurely. The majority of preterm births in our study were spontaneous and not due to iatrogenic reasons. This concurs with prior studies, demonstrating a higher trend of preterm births among pregnant women with COVID-19 [13]. We also observed a relatively high rate of hypertensive disorders of pregnancy $(13.4 \%)$. It is possible that placental-mediated insult due to COVID-19 related coagulopathy may have a role in this finding $[17,18]$. Further studies are warranted to investigate the mechanisms underlying these adverse outcomes.

As universal testing for COVID-19 was not implemented in all participating centers, it is possible that additional asymptomatic SARS-CoV-2-infected women who have given during the study period were not captured. In addition, we cannot exclude the possibility that the relatively low rate of comorbidities in the current cohort could have contributed to the mild course of disease in most women. It is plausible that a higher rate of cesarean delivery would have been encountered in populations with more prevalent comorbid conditions and different disease course severities. Finally, serologic testing was not carried among neonates. The strengths of the current study include its prospective design and the meticulous data collection.

In conclusion, in this study, among SARS-CoV-2-infected mothers, vaginal delivery rates were high and associated with favorable outcomes with no cases of neonatal COVID19. Our findings underscore and support current recommendations to manage deliveries among SARS-CoV-2-infected mothers on the basis of obstetric indications. Future studies are warranted to confirm our findings and better delineate the role of vertical transmission in the setting of maternal COVID-19.

Author contributions AR, BR, DK, SP, NB, YO, SB, HAK, AW, YK, RM, SJL, UA, TBS, GC, YST, IBS, and YY reviewed the literature and wrote the paper. AR performed the statistical analyses for this study. AT and YY designed the prospective data collection and constructed the REDCap database. All authors read and approved the final manuscript.

Funding The study was funded by the Israeli Ministry of Science and Technology.

\section{Compliance with ethical standards}

Conflict of interest The authors declare that they have no conflicts of interest.

Ethical approval The study was approved in March 2020 by all participating centers. For this type of study, formal consent was not required and was waived by the institutional review board approval. All procedures performed in studies involving human participants were in accordance with the ethical standards of the institutional and/ or national research committee and with the 1964 Helsinki declaration and its later amendments or comparable ethical standards.

\section{References}

1. World Health Organization. WHO Director-General's opening remarks at the Mission briefing on COVID-19 - 12 March 2020. https://www.who.int/zh/dg/speeches/detail/who-director-generals-opening-remarksat-the-mission-briefing-on-covid-19. Accessed 12-march-2020

2. Breslin N, Baptiste C, Gyamfi-Bannerman C, Miller R, Martinez $\mathrm{R}$, Bernstein K et al (2020) COVID-19 infection among asymptomatic and symptomatic pregnant women: two weeks of confirmed presentations to an affiliated pair of New York City hospitals. Am J Obstet Gynecol MFM 2(2):100118

3. Yu N, Li W, Kang Q, Xiong Z, Wang S, Lin X, Liu Y, Xiao J, Liu H, Deng D, Chen S, Zeng W, Feng L, Wu J (2020) Clinical features and obstetric and neonatal outcomes of pregnant patients with COVID-19 in Wuhan, China: a retrospective, single-centre, descriptive study. Lancet Infect Dis 20(5):559-564

4. Mehan A, Venkatesh A, Girish M (2020) COVID-19 in pregnancy: risk of adverse neonatal outcomes. J Med Virol

5. Dong L, Tian J, He S, Zhu C, Wang J, Liu C et al (2020) Possible vertical transmission of SARS-CoV-2 from an infected mother to her newborn. JAMA 323(18):1846-1848

6. Penfield CA, Brubaker SG, Limaye MA et al (2020) Detection of SARS-COV-2 in placental and fetal membrane samples. Am J Obstet Gynecol MFM 2:100133

7. Egloff C, Vauloup-Fellous C, Picone O, Mandelbrot L, Roques $\mathrm{P}$ (2020) Evidence and possible mechanisms of rare maternal-fetal transmission of SARS-CoV-2. J Clin Virol 128:104447

8. Patanè L, Morotti D, Giunta MR, Sigismondi C, Piccoli MG, Frigerio L et al (2020) Vertical transmission of COVID-19: SARS-CoV-2 RNA on the fetal side of the placenta in pregnancies 
with COVID-19 positive mothers and neonates at birth. Am J Obstet Gynecol MFM 2(3):100145

9. Chatzistamatiou K, Sotiriadis A, Agorastos T (2016) Effect of mode of delivery on vertical human papillomavirus transmission - a meta-analysis. J Obstet Gynaecol 36:10-14

10. Simonsen KA, Anderson-Berry AL, Delair SF, Davies HD (2014) Early-onset neonatal Sepsis. Clin Microbiol Rev 27:21-47

11. Coronavirus (COVID-19) infection and pregnancy. Royal College of Obstetricians \& Gynaecologists; 2020 n.d. https://www.rcog. org.uk/en/guidelinesresearchguidelinesresearch-services/guide lines/coronavirus-pregnancy/ Accessed July 7, 2020

12. Novel Coronavirus 2019 (COVID-19). American College of Obstetricians and Gynecologists; 2020 n.d. https://www.acog. org/clinical/clinical-guidance/practice-advisory/articles/2020/03/ novel-coronavirus-2019 Accessed July 7, 2020

13. Matar R, Alrahmani L, Monzer N, Debiane LG, Berbari E, Fares J, Fitzpatrick F, Murad MH (2020) Clinical presentation and outcomes of pregnant women with COVID-19: a systematic review and meta-analysis. Clin Infect Dis

14. Prabhu M, Cagino K, Matthews KC, Friedlander RL, Glynn SM, Kubiak JM, Yang YJ, Zhao Z, Baergen RN, DiPace JI, Razavi AS, Skupski DW, Snyder JR, Singh HK, Kalish RB, Oxford CM, Riley
LE (2020) Pregnancy and postpartum outcomes in a universally tested population for SARS-CoV-2 in New York City: a prospective cohort study. BJOG 127:1548

15. Wu Z, McGoogan JM (2020) Characteristics of and important lessons from the coronavirus disease 2019 (COVID-19) outbreak in China: summary of a report of 72314 cases from the Chinese center for disease control and prevention. JAMA 323(13):1239-1242

16. Knight M, Bunch K, Vousden N, Morris E, Simpson N, Gale C (2020) Characteristics and outcomes of pregnant women admitted to hospital with confirmed SARS-CoV-2 infection in UK: national population based cohort study. BMJ 369:m2107

17. Becker RC (2020) COVID-19 update: Covid-19-associated coagulopathy. J Thromb Thrombolysis 50(1):54-67

18. Shanes ED, Mithal LB, Otero S et al (2020) Placental pathology in COVID-19. Am J Clin Pathol 154:23-32

Publisher's Note Springer Nature remains neutral with regard to jurisdictional claims in published maps and institutional affiliations. 